\title{
Propagation and Active Control of Femtosecond Plasmon Pulses
}

\author{
Z. L. Sámson ${ }^{1}$, K. F. MacDonald ${ }^{1}$, P. Horak ${ }^{1}$, M. I. Stockman' ${ }^{2}$, and N. I. Zheludev ${ }^{1}$ \\ 1. Optoelectronics Research Centre, University of Southampton, Highfield, Southampton, Hampshire, SO17 1BJ, UK \\ 2. Department of Physics and Astronomy, Georgia State University, 29 Peachtree Center Avenue, Atlanta, GA 30303-4106, USA
}

Here we report on the ultrafast modulation of propagating fs SPP signal via direct optical excitation of the metal component of an aluminium/silica waveguide and investigate spectral dependence of the effect and its kinetics. We also analyse numerically the nonlinear SPP pulse propagation regimes, including self-focusing, pulse compression and solitons, supported by the nonlinearity of the metal component of the waveguide.

Since the term 'active plasmonics' was coined in 2004, techniques based on a wide variety of 'switchable' media, including thermo- and electro-optic materials, quantum dots, and photochromic molecules, have been developed to control the propagation of guided surface plasmon-polariton (SPP) signals. Recent years have also seen increasing interest in ultrafast and nonlinear plasmonic phenomena [1]. In this work we bring together the fields of active plasmonics and ultrafast/nonlinear optics, considering linear and nonlinear modes of femtosecond SPP pulse propagation, and describing recent work on the ultrafast active modulation of SPP pulses.

The ultrafast modulation of a propagating fs SPP signal (Fig. 1) has been demonstrated recently in ref. 2. This modulation scheme provides switching speeds several orders of magnitude faster than other techniques with the additional advantage that it does not require dedicated 'active' media. We report here on recent experiments revealing the effect's strong dependence on wavelength in the vicinity of Al's interband transition at $\sim 800 \mathrm{~nm}$.

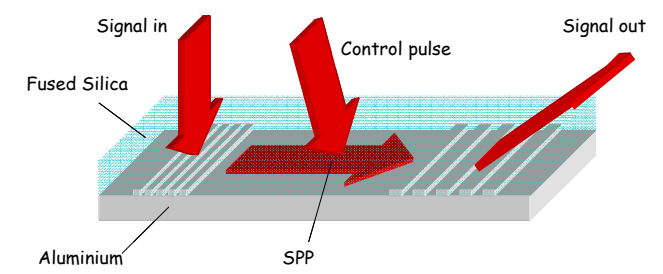

Fig. 1 Femtosecond experiment on controlling plasmon signals: A plasmonic signal, coupled to and from an aluminium/silica waveguide by gratings, is modulated by fs optical control pulses as it travels between the gratings.

SPPs have great potential as information carriers for next-generation, highly integrated nanophotonic devices but our analysis shows that care must be taken in the short pulse regime, where group velocity and loss dispersion can lead to substantial pulse re-shaping during propagation (Fig. 2), with obvious implications for the achievable repetition rate and signal contrast in device applications.

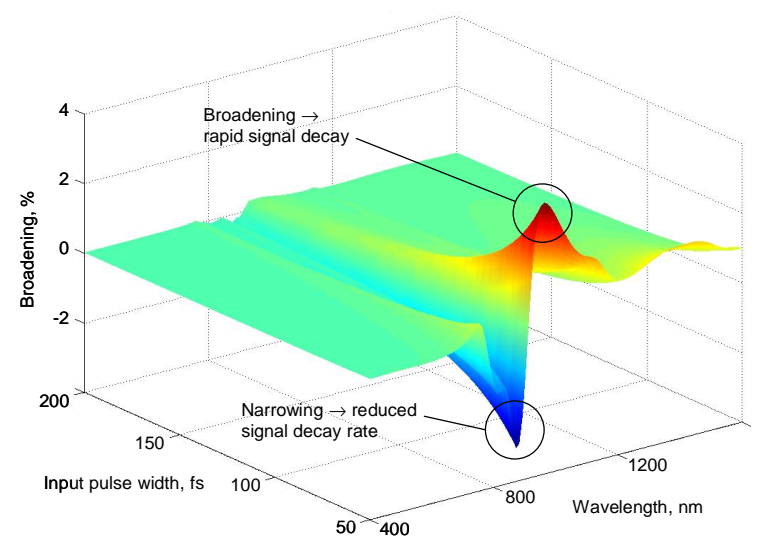

Fig. 2 Percentage SPP pulse broadening per decay length of propagation as a function of wavelength and input pulse duration for an aluminium/silica waveguide.

These results invite broader questions as to what can ultimately be achieved in nonlinear and active plasmonics. Here we analyse the possibility of nonlinear SPP pulse propagation regimes, including self-focusing, pulse compression and solitons, supported by the nonlinearity of the metal component of the waveguide.

\section{References}

[1] L. Cao, and M. L. Brongersma, "Active Plasmonics: Ultrafast developments," Nat. Photon. 3, 12 (2009).

[2] K. F. MacDonald, Z. L. Sámson, M. I. Stockman, and N. I. Zheludev, "Ultrafast active plasmonics," Nat. Photon. 3, 55 (2008). 